\title{
The missing element
}

Investigating the prevalence of non-fluoride toothpaste use in adults and children using nationally representative data from New Zealand: a crosssectional study. Br Dent J 2020; 228: 269-276. https://doi.org/10.1038/s41415-020-1304-5

In May 1955, dentist and biochemist Joseph C. Mulher wrote 'All of us who are interested in dental health are anxious to find effective anticariogenic agents' and 'I will continue to work hard on the solution of the dental caries problem until a satisfactory product is available. ${ }^{1} \mathrm{He}$ would later introduce what is regarded as the first commercially available fluoride-containing toothpaste. ${ }^{2}$ Fast forward sixty-five years, and the consumer market is flooded with an array of fluoride-containing toothpastes and mouthwashes as the importance of this substance in preventing dental decay is now common knowledge among the general public. Overwhelmingly, dental

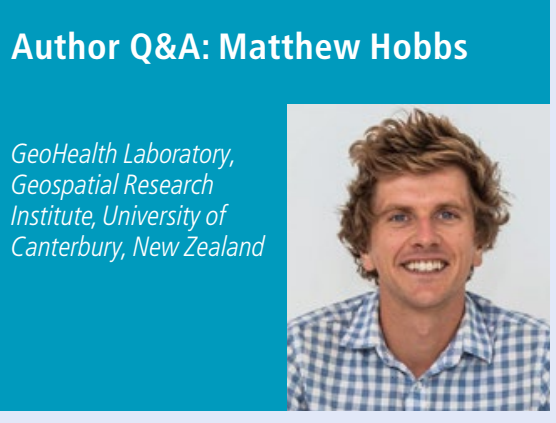

Why did you decide to undertake this study?

We saw anecdotal evidence from the New Zealand Dental Association (NZDA) and British Dental Association (BDA) that the rise of non-fluoride or natural toothpastes pose a threat to good oral health. However, it soon became clear there was a lack of contemporary evidence internationally that used large nationally representative samples to examine the prevalence of nonfluoride toothpaste use in both adults and children.

\section{Did any of the results surprise you?}

Two results stood out. First, we did not expect the prevalence to be so high. Approximately one in fifteen adults and children use non-fluoride toothpaste in New Zealand. This is concerning as a recent review showed that personal oral hygiene in the absence of fluorides fails to show a professionals recognise years of robust research confirming fluoride toothpaste use in children and adults as a key strategy in the prevention of caries. Strengthening this position, in 2019 a Cochrane Review ${ }^{3}$ showed that oral hygiene in the absence of fluoride fails to show a benefit in reducing the incidence of dental caries.

With this in mind, a recent study carried out at the University of Canterbury (NZ) analysed self-reported data from the New Zealand Health Survey of 2017/18 to investigate the prevalence of nonfluoride toothpaste use. Corresponding sociodemographic and socioeconomic factors were explored revealing some interesting

benefit in terms of reducing the incidence of dental caries. Second, in health-related research, we often see a social gradient in health, such that, those in the most deprived areas exhibit the unhealthy behaviours and poorer health relative to those most affluent areas. In contrast, this study showed that the highest prevalence of non-fluoride toothpaste use was in the moderately and the least deprived areas. Moreover, for children, those in the most deprived areas had the lowest use of non-fluoride toothpaste. This highlights the complexity of dental healthrelated behaviours. They are certainly not as simple as we first thought and require some careful thought around intervention.

What do you think the next steps should be considering your findings?

Oral health promotion strategies involving fluoride, such as fluoride toothpaste, have consistently been shown to provide benefit and are supported by high-quality evidence. It will be important for public health to translate these findings into policy to improve population health. We all need to be aware of the benefits of using fluoride and the risks of using nonfluoride or naturally marketed toothpastes. This is especially important in an era of miscommunication, fake news, and clever marketing techniques. results. Overall, $6.8 \%$ of adults and $6.4 \%$ of children reported to use non-fluoride toothpaste. Individuals identifying as Asian by ethnicity were most likely to report use of non-fluoride toothpaste. Perhaps surprisingly, the nationally representative data showed that the highest prevalence of non-fluoride toothpaste use was seen in areas defined as moderately and least deprived. As such, any health intervention leading to a decline in non-fluoride toothpaste use may widen already existing health inequalities. The results hereby highlight the complexity of dental health-related behaviours and raise questions regarding the targeting and funding of public health initiatives.

So why might almost $7 \%$ of the NZ population be using a non-fluoride toothpaste? A quick internet search of fluoride - alone or in relation to oral health - immediately unearths somewhat dubious claims of its numerous health 'hazards'. It doesn't take long to stumble across outlandish comments, conspiracy theories and controversies surrounding fluoridation. Or might consumers now be more interested in the cosmetic benefits of toothpastes for example, whitening, rather than simply preventing decay and so accept low or zero fluoride content? Considering the global burden of disease relating to caries (estimated at affecting 2.4 billion people worldwide), ${ }^{4}$ it would seem imperative to continue efforts to promote and facilitate the use of fluoride toothpaste in the general population.

\section{By Anne C. Devlin Dundee Dental Hospital, NHS Tayside}

\section{References}

1. Muhler J C. Stannous Fluoride Dentifrice. J Am Dent Assoc 1955; 50: 578.

2. Lippert F. An Introduction to Toothpaste - Its Purpose, History and Ingredients. In Van Loveren C (ed) Toothpastes. pp.1-14. Monogr Oral Sci. Basel, Karger, 2013.

3. Walsh T, Worthington H, Glenny A, et al. Fluoride toothpastes of different concentrations for preventing dental caries. Cochrane Database Syst Rev 2019: DOI: 10.1002/14651858.CD007868.pub3.

4. Petersen P E. The World Oral Health Report 2003: Continuous improvement of oral health in the 21st Century - The approach of the WHO Global Oral Health Programme. Community Dent Oral Epidemiol 2003; 31 Suppl 1: 3-23. 